\title{
Study of a Cost-effective Localization Algorithm in Wireless Sensor Networks ${ }^{\star}$
}

\author{
Xin Li, Bei Hua ${ }^{\star \star \star}$, and Yan Guo \\ Department of Computer Science and Technology \\ University of Science and Technology of China, Anhui, Hefei 230027, China \\ Mobile Computing Laboratory \\ Suzhou Institute for Advanced Study, Suzhou, Jiangsu 215123, China \\ xinxinol@mail.ustc.edu.cn, bhua@ustc.edu.cn, guoyan6@mail.ustc.edu.cn
}

\begin{abstract}
Currently, most of the RSS-based localization approaches rely on an isotropic radio propagation model to compute the distance from RSS, which however has been proved to be impossible by recent research work. Performance analysis is necessary to evaluate the applicability of a localization algorithm, however, few work has been done to evaluate and compare the performance of existing localization algorithms in simulated realistic settings. This paper first investigates the relationship between distance and RSS for symmetric and asymmetric links based on a realistic radio propagation model; and then provide the detail implementation of a RSS-based approach called LSBA; finally gives a panorama of performance comparison of LSBA and other four localization algorithms in terms of estimation error, convergence speed, computational complexity and communication cost in the simulated realistic environment. Simulation results show that LSBA achieves the best trade off among all the four metrics in networks with moderate number of anchors, and has good adaptability to irregular node deployment.
\end{abstract}

Key words: RSS; wireless sensor networks; localization

\section{Introduction}

Localization approaches are roughly classified as fine-grained approaches and coarse-grained approaches. Fine-grained approaches normally require accurate distance or angle measurements to compute the location of unknown node. TDOA (Time Difference of Arrival) [7] [8] [9], AOA (Angle of Arrival) [10], and RIPS (Radio Interferometric Positioning System) [12] rely on extra (sometimes expensive and complex) hardware other than radio transceiver to get accurate measurements. Use of RSS as ranging technique receives much recognition since radio transceiver is the only available ranging device for most of the common sensor nodes. Most of the existing RSS methods rely on an ideal radio propagation

\footnotetext{
* This work was supported by the National Natural Science Foundation of China under Grant No.60673173.

${ }^{\star \star \star}$ to whom correspondence should be addressed.
} 
model [16] to get the distance from RSSI, which states that the received signal strength diminishes with the distance according to certain law. However, recent researches [13] [14] [15] show that radio propagation pattern is highly random in real world, and no one-to-one mapping exists between RSSI and distance in most situations. Therefore localization algorithms based on ideal radio model may perform poorly in realistic environment, and need to be reconsidered. Coarse-grained approaches normally rely on proximity and near-far information or less accurate distance estimation to infer the location of unknown node, of which Centroid [2], geometry constrains [6], DV-HOP [4], Amorphous [5], LSBA (Link State Based Annulus localization algorithm) [17] are typical. Coarse-grained approaches are much popular in densely deployed large scale sensor networks since they avoid the difficulty of getting accurate measurements; however their performance may degrade due to inaccurate information and need careful evaluation.

Performance of a localization algorithm can be measured by various metrics, in which estimation error, un-localizable ratio, convergence speed, computational and communication complexity are the most important ones. Average estimation error is a classical performance metric that measures the average localization accuracy. Un-localizable ratio is the ratio of nodes that cannot be localized even after certain rounds of localization, which can be used to depict how fast the process winds up with stable estimation error. Since wireless sensor nodes are tiny devices with constrained computing ability, storage, bandwidth, and energy, computational complexity must be taken into account. The requirement of energy saving is usually translated into lowering the communication complexity, since most of the energy is consumed by communication in WSN. However, most of the previous work only take one or two of the performance metrics into consideration, such as, [2] [4] [5] and [6] only focus on estimation accuracy; [3] and [17] focus on estimation accuracy, communication cost or un-localizable ratio. In this paper, we provide a comprehensive performance comparison of five algorithms in terms of the four performance metrics in realistic experimental settings.

The remainder of this paper is organized as follows: section 2 investigate the relationship between distance and RSSI for symmetric link and asymmetric link based on a realistic radio propagation model; section 3 describes the design and implementation of LSBA; section 4 compare LSBA with Centroid [2], APIT [3], DV-HOP [4], and Amorphous [5] in various performance metrics in realistic experimental settings; and section 5 concludes.

\section{Motivation}

Our work is largely inspired by [14], where a Radio Irregularity Model (RIM) is brought up based on empirical data obtained from MICA2 platform to characterize the radio pattern in real wireless sensor networks. Radio irregularity is mainly caused by heterogeneous sending powers and anisotropic path losses, and is modeled as follows:

$$
R S S=S P \times(1+R \times V S P)-P L \times K_{i}+X
$$


The first part of (1) accounts for the difference in hardware calibration and battery status, where SP (Sending Power) is the power rating of node, VSP (Variance of Sending Power) is defined as the maximum percentage variance of signal sending power among different devices, and $\mathrm{R}$ is a normal random variable that measures the variance caused by hardware. The second part reflects the anisotropism of radio, where PL is the free space loss, and $K_{i}$ represents the difference in path loss in different directions.

$$
\begin{aligned}
& K_{i}=\left\{\begin{array}{l}
1, i=0 \\
K_{i-1} \pm \text { Rand } \times D O I, 0<i<360 \wedge i \in N
\end{array}\right. \\
& \text { where }\left|K_{0}-K_{359}\right| \leq D O I
\end{aligned}
$$

$K_{i}$ is calculated according to (2), where DOI (Degree of Irregularity) is defined as the maximum range variation per unit degree, and Rand is a Weibull distribution random variable. The third part of (1) models the environment noise that follows normal distribution. More details can be found in [14].

Experiments show that lots of asymmetric links exist in network due to radio irregularity, and the number of asymmetric (symmetric) links increases (decreases) with longer distance. This observation inspired us to exploit link information to reduce the distance uncertainty between a pair of nodes. To validate our imagination, we simulated with RIM model in various parameter settings and got two curves on RSS vs. distance for symmetry links and asymmetry links respectively. Fig. 1 was gained with $\mathrm{SP}=-5 \mathrm{dBm}, \mathrm{VSP}=0.1, \mathrm{DOI}=0.002$, Weibull $=[0.16,0.67]$, and receiver threshold $=-70 \mathrm{dBm}$.

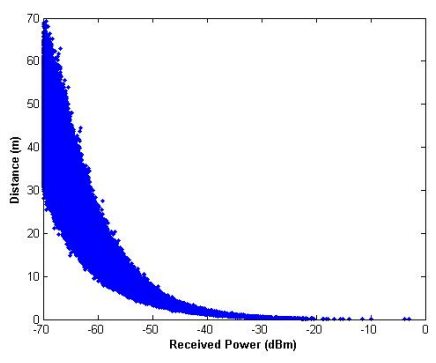

(a) Symmetry link

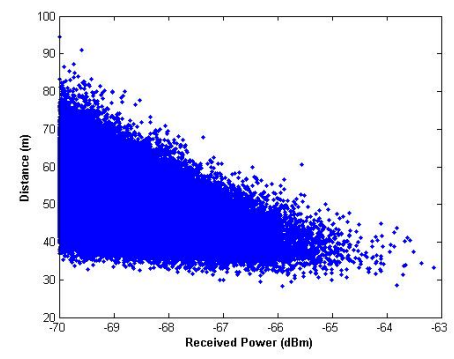

(b) Asymmetry link

Fig. 1. RSS vs. Distance

The curves suggest that rough relationships exist between RSSI and range of distance when link type is given. This result motivated us to design LSBA that makes use of the link type and RSSI to refine the possible areas an unknown node may reside in, and then calculates the centroid of the overlapping area as the location. Since the curves of RSS vs. Distance vary with network settings and environment, they need to be recomputed for each concrete situation. Nevertheless, it will not add much difficulty, since all the device related parameters such as SP, VSP and receiver threshold can be easily obtained from devices, 
and the environmental parameters such as DOI and Weibull distribution can be obtained from empirical values or measured in real environment.

\section{Design and Implementation of LSBA}

The basic idea of LSBA is as follows: each unknown node exchanges messages with its location-aware neighbors to find out the type of link (symmetric or asymmetric) between itself and each of these neighbors; then calculates the range of distance to each neighbor based on the type and RSSI of each link; after getting all the distance ranges, draws an annulus for each link centering at the locationaware node with the radius as the corresponding distance range; finally calculates the centroid of the highest overlapping area as its location estimation. Therefore LSBA consists of two steps: link state construction and location calculation, in which a link state exchange protocol and a grid-based method are provided to simplify the calculation of the highest overlapping area of annuli (fig.2(a)).

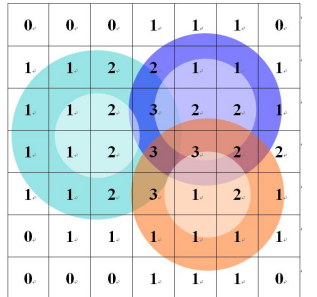

(a) Annuli Drawing

\begin{tabular}{|c|c|c|c|c|c|}
\hline RSSI $(\mathrm{dBm})$ & -5 & $\ldots$ & -60 & $\ldots$ & -70 \\
\hline \hline Min $(\mathrm{m})$ & 0.0000 & $\ldots$ & 10.0129 & $\ldots$ & 28.9374 \\
\hline Max $(\mathrm{m})$ & 0.0000 & $\ldots$ & 30.3575 & $\ldots$ & 70.1853 \\
\hline
\end{tabular}

(b) RSS vs. Distance lookup table of fig.1(a)

Fig. 2. Implementation of LSBA

The link state exchange protocol works as follows. Each node broadcasts its ID and location (if it's a location-aware node) in an advertisement message. After receiving an advertisement, a node records the sender and RSSI of the message and puts [sender, RSSI] in its Asymmetry-RSSI-Array, then calculates the distance range (expressed as a [min, max] pair) to the sender using the curve for asymmetric link and puts [sender, [min, max]] in the AsymmetricDistance-Array, at last sends back a response message containing the sender and the intended receiver. If an asymmetric link exists between a pair of nodes, say from A to B, then only B receives A's advertisement and A cannot hear B, and the message exchange between $\mathrm{A}$ and $\mathrm{B}$ ends at this point. If a symmetric link exists between a pair of nodes, then either side can receive the advertisement and response message of the other side. After receiving a response message that replies to its previous advertisement, a node removes the corresponding item of [sender, RSSI] from the Asymmetry-RSSI-Array to the Symmetry-RSSI-Array, and deletes the corresponding item of [sender, [min, max]] from the AsymmetricDistance-Array, then recalculates the distance range using the curve for symmetric link and puts the new [sender, [min, max]] in the Symmetric-Distance-Array, at last sends back a distance notification message containing the distance range 
it calculates. The exchange of distance range is to further reduce the uncertainty of distance between a pair of nodes. Therefore after receiving a distance notification, the unknown node takes the intersection of the two distance ranges as the final distance range. The current link state exchange protocol doesn't take into account collision and packet lost.

To simplify the computation of distance range, we use two lookup tables calculated offline to replace the two RSS vs. Distance curves. Each table is organized as an array, where $\mathrm{N}$ is the size of the table and each entry records the [min, max] pair of a RSSI. As an example, fig.2(b) approximates the curve in fig.1(a) with the range of RSSI from $-5 \mathrm{dBm}$ to $70 \mathrm{dBm}$ and a step size of $1 \mathrm{dBm}$. For a non-integral RSSI, the nearest integral RSSI is taken.

If there are only a few anchors in the network, many nodes may remain unknown after the first round of localization. In the following rounds, all locationaware nodes, including anchors and those getting their locations in the previous round, broadcast their locations, and non-anchor nodes either locate themselves or refine the locations obtained in the previous rounds. This process repeats until the algorithm converges. The decision of when to stop is made in a distributed way. When a non-anchor node finds that the variance of its location estimation is below a threshold, it stops updating its location and stops broadcasting advertisement, but it can still respond to other node's advertisement. When all the nodes cease broadcasting advertisement, the localization process winds up.

\section{Performance Evaluation}

We simulated Centroid, APIT, DV-HOP, Amorphous and LSBA on Matlab and compared them in terms of estimation error, residual ratio of un-localized nodes, communication complexity, and computational complexity in realistic simulation environment. Estimation error is defined as the average variance between estimated location and real location. Residual ratio of un-localized nodes (called residual ratio for short) is the ratio of un-localized nodes after certain rounds of localization, which can be used to reflect the convergence speed as well as the localization ability of algorithm. Communication and computational complexity are defined respectively as the total number of packets exchanged for and the total amount of time spent on localization.

Network settings generally have great influence on performance of localization algorithms, among which average connectivity (AC), number of anchors $(\mathrm{AN})$, and distribution of nodes (ND) are the most important ones. Average connectivity is defined as the average number of neighbors per node, and is an indicator of network density. Evenly deployed network is assumed by many localization algorithms, yet it is hard to achieve in real world. The adaptability to irregular node distribution is crucial to the robustness of algorithm. To evaluate these algorithms in a realistic simulation environment, we use RIM model to model the radio propagation pattern, and change the radio irregularity via DOI and VSP. The values of DOI, VSP, Weibull and the environment noise are all from [14], which is obtained from the empirical data. 
We conducted the experiments on Matlab 7.0.4 that runs on laptop IBM ThinkPad R51BC1, and omit the detailed implementation of the other algorithms due to limitation of space. For ease of comparison we didn't include iterative localization refinement in our experiments, since no such process exists in DV-HOP and Amorphous, and it greatly increases the computational complexity of APIT. For communication complexity, we didn't take collision, packet lost and traffic control into account. For computational complexity, we only counted the time spent on localization related calculations, and the computation methods are derived from APIs of Matlab without any modification.

In the following experiments, without specification, sensors (include anchors) are uniformly distributed in an area of $300 \times 300 \mathrm{~m}^{2}$; the free space propagation radius of sensors is $48.9 \mathrm{~m}$; the value of DOI and VSP are 0.002 and 0.1 respectively; the value of Weibull is $[0.16,0.67]$. Each experiment was run 500 times with different random seeds to get the performance.

\subsection{The Influence of Average Connectivity}

In this experiment, we investigate the influence of average connectivity $(\mathrm{AC})$ on algorithm performance with $A N=36$.

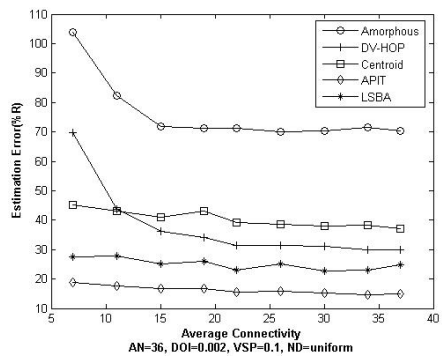

(a) Estimation Error

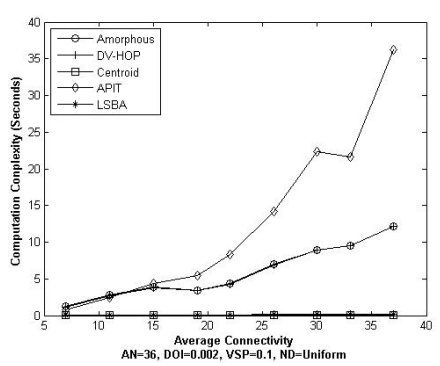

(c) Computation Complexity

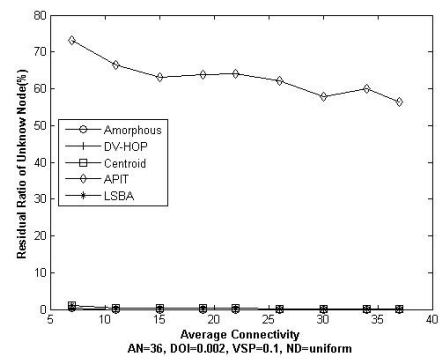

(b) Residual Ratio

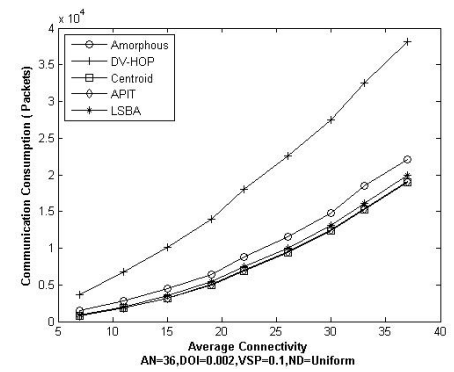

(d) Communication Cost

Fig. 3. The Influence of Average Connectivity

In fig.3(a), APIT has the lowest estimation error with LSBA following on the heels of it. In fig.3(b), the residual ratio of APIT is above $50 \%$ after two rounds of localization, yet the residual ratio of other algorithms almost reach 0 . 
This is due to the stringent localizable constraints in APIT that each localizable node must reside in at least one triangle formed by three location-aware neighbors, whereas other algorithms have much relaxed constraints. Similar results are observed in other experiments indicating that APIT has the lowest convergence speed. In fig.3(c), the computation time of APIT increases most rapidly, since larger AC brings more nodes to participate in the PIT test; DV-HOP and Amorphous nearly overlap and grow quickly as well, this is because they use the same estimation methods; Centroid and LSBA have the lowest computation complexity. In fig.3(d), communication complexity of DV-HOP and Amorphous increase most rapidly and DV-HOP consumes more packets than Amorphous, since they need more packets to construct routing tables and DV-HOP has to broadcast Hop-Size whereas Amorphous computes it offline.

DV-HOP and Amorphous can get better estimation accuracy at higher AC, which also leads to higher communication cost; APIT achieves the best estimation accuracy, but its residual ratio and computational complexity is the highest; LSBA and Centroid have the lowest residual ratio, communication and computational complexity, and LSBA has better estimation accuracy than Centroid.

\subsection{The Influence of Number of Anchors}

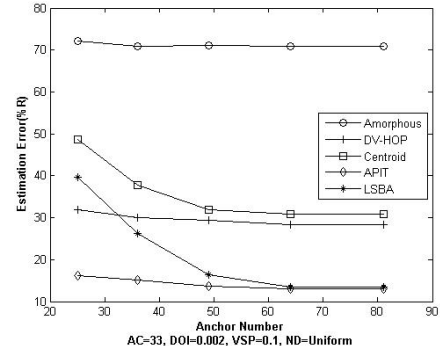

(a) Estimation Error

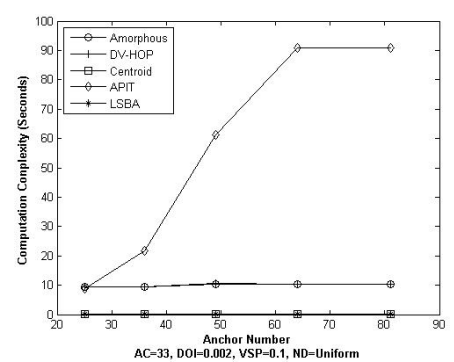

(c) Computation Complexity

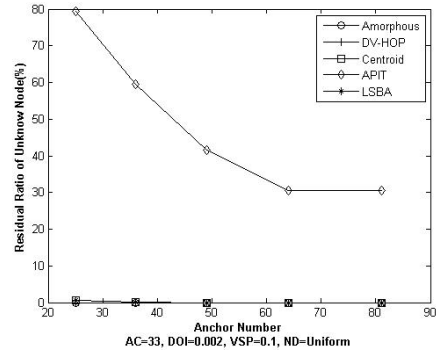

(b) Residual Ratio

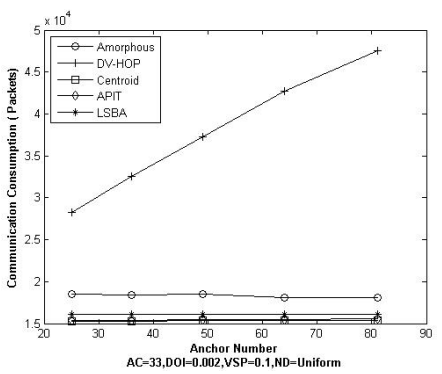

(d) Communication Cost

Fig. 4. The Influence of Number of Anchors

In this experiment, we study the influence of number of anchors on algorithm performance. Since DV-HOP and Amorphous get better estimation accuracy at 
high $\mathrm{AC}$ while other algorithms are not sensitive to it, we choose to conduct this experiment with $A C=33$.

In fig.4(a), DV-HOP, Amorphous and APIT are not sensitive to the number of anchors. Estimation error of Centroid and LSBA decrease rapidly when number of anchors increases, and LSBA performs much better than Centroid as it makes use of the link information. Although APIT achieves the highest location accuracy with only a few anchors, more anchors will greatly improve its convergence speed, see fig.4(b). In fig.4(c), the computation time of APIT increases rapidly when the number of anchors increases, since more anchors appearing in the neighborhood of an unknown node increase the number of PIT tests. Other curves hardly change, since the amount of nodes in the network doesn't change when average connectivity is fixed, and moreover the number of unknown nodes decreases when the number of anchors increases. In fig.4(d), the communication cost of DV-HOP is the highest, and moreover it increases rapidly with the number of anchors, since more anchors require more broadcast of Hop-Size.

To sum up, if only a few anchors exist in the network, DV-HOP and APIT are better choices due to their lower estimation error; however when more anchors are available, LSBA is preferable as it achieves the best tradeoff between localization accuracy and energy saving.

\subsection{The Influence of DOI}

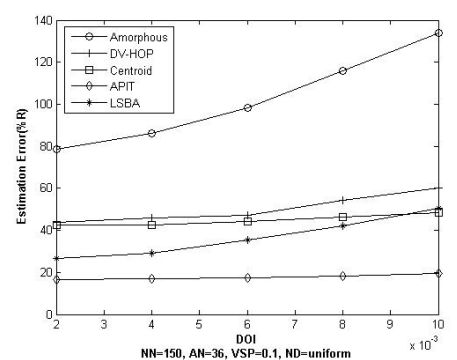

(a) Estimation Error

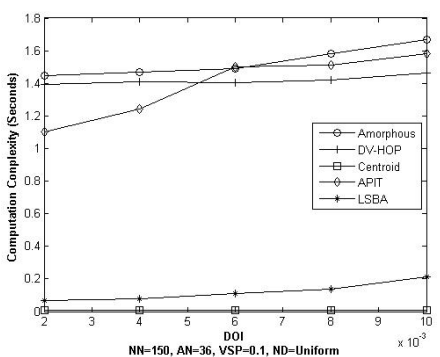

(c) Computation Complexity

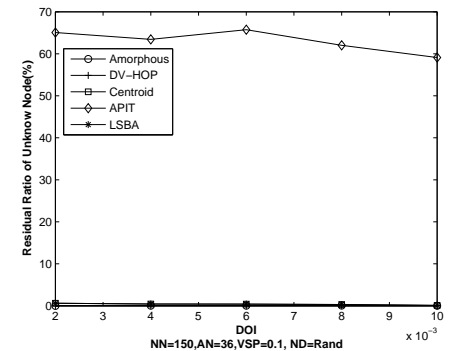

(b) Residual Ratio

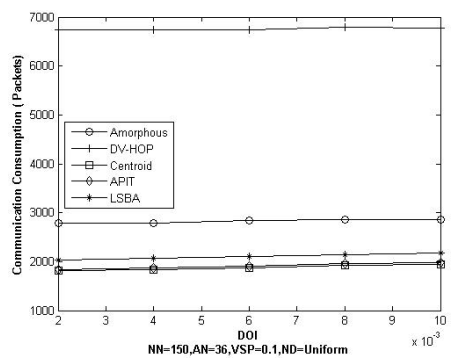

(d) Communication Cost

Fig. 5. The Influence of DOI 
In this experiment, we study the influence of DOI on algorithm performance. Since average connectivity changes with DOI, we choose to fix the number of nodes (NN) instead of average connectivity in this experiment. In this case, the average connectivity is around 7 that is the most common situation in real world.

It's easy to know from formula 1 that DOI has smaller influence on RSS than VSP as it changes from 0.002 to 0.01 . Nevertheless the estimation error of Amorphous is affected greatly by DOI, since its Hop-Size is calculated based on average connectivity that is influenced by DOI. The estimation error of LSBA also increases, as increased DOI enlarges the distance uncertainty. However, the estimation error of Centroid and APIT hardly change, as they only care about the locations of heard anchors, meanwhile the number of heard anchors is less affected by DOI. In fig.5(a), the computation time of DV-HOP and Amorphous is very high, because irregular signal pattern causes more inaccurate distance estimation which in turn takes the estimator more time to search the optimal solution. In fig.5(d), the communication cost of all the algorithms are not sensitive to DOI, in which DV-HOP is the highest.

\subsection{The Influence of VSP}

In this experiment, we study the influence of VSP on algorithm performance. Since average connectivity also changes with VSP, we fix the number of nodes instead of average connectivity in the experiment.

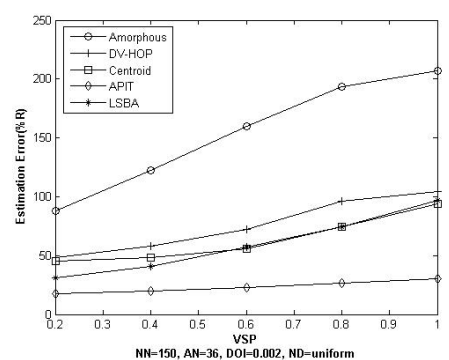

(a) Estimation Error

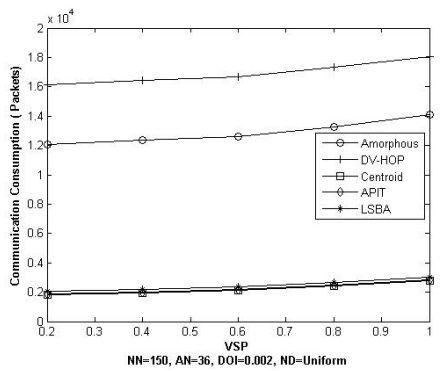

(c) Computation Complexity

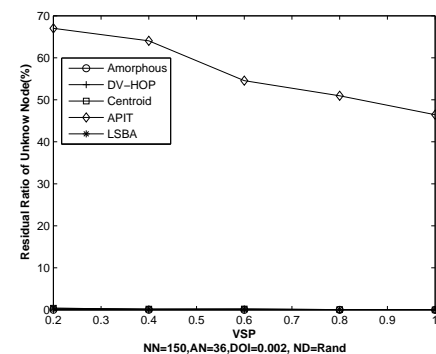

(b) Residual Ratio

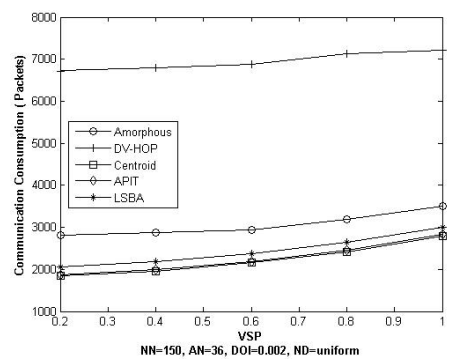

(d) Communication Cost

Fig. 6. The Influence of VSP 
Compared with DOI, VSP has much greater contribution to radio irregularity when it changes from 0.2 to 1 , so nearly all the algorithms experience increased estimation error in fig.6(a). An interesting observation is that increased VSP helps to reduce the residual ratio of APIT (fig.6(b)). Similar to fig.5(c), the computational complexity of DV-HOP and Amorphous in fig.6(c) is very high due to the large number of inaccurate distance estimation caused by increased VSP. Another observation in fig.6(c) is that the computation time of APIT increases very fast than that in fig.5(c), the reason may be that highly irregular radio pattern brings more nodes to the neighborhood of an unknown node, and thus increases the number of PIT tests. This also explains why increased VSP reduces the residual ratio of APIT. Similar to section 4.3, communication cost of DV-HOP is still the highest in fig.6(d).

\subsection{The Convergence Speed of APIT}

Above experiments show that although APIT has the highest estimation accuracy, its convergence speed is very low. In this section, we investigate the convergence characteristic of APIT using the same environment setting as that in section 4.1.

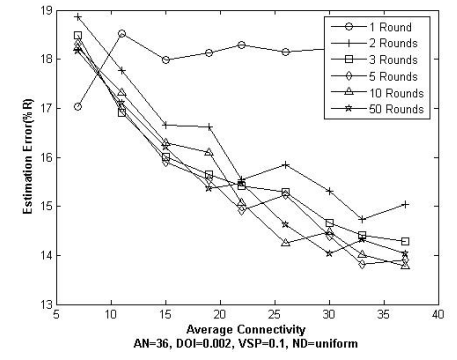

(a) Estimation Error

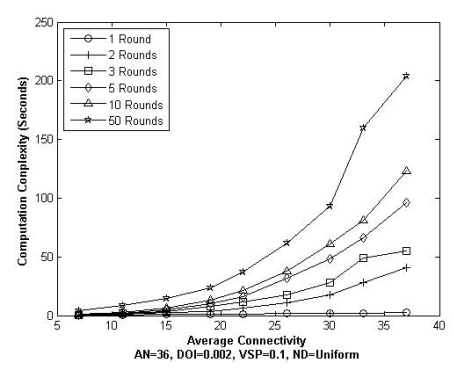

(c) Computation Complexity

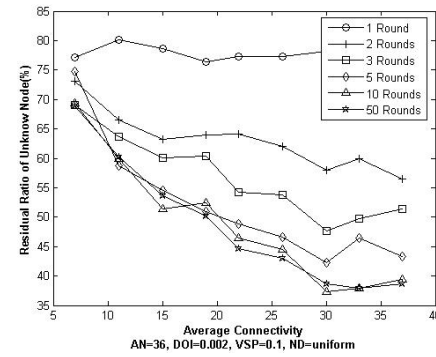

(b) Residual Ratio

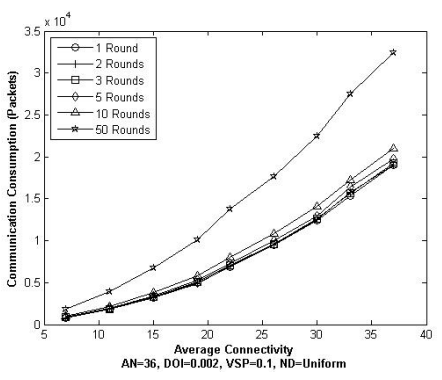

(d) Communication Cost

Fig. 7. The Convergence Speed of APIT

From fig.7, both the estimation error and residual ratio decrease rapidly in the first three rounds, and then decline very slowly; meanwhile the computation and communication cost increase steadily. The residual ratio is as high as $35 \%$ 
after tens of rounds even under high $\mathrm{AV}$, the reason is that the nodes near the boundary as well as those in regularly deployed area cannot find three neighbors that can hold it in a triangle. Therefore mobile anchors are needed to help APIT improve the convergence speed and localizable ratio.

\subsection{Performance in C-Shaped Area}

In this experiment we study the adaptivity of algorithms to irregular node deployment. We conduct the experiment in a C-shaped area that is limited in a square with $\mathrm{AC}$ increasing from 7 to 70 , see fig.8(a). In this section we only focus on the estimation error, since others are much similar to that in section 4.1.

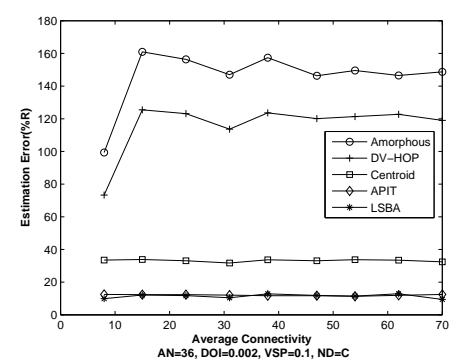

(a) Estimation Error

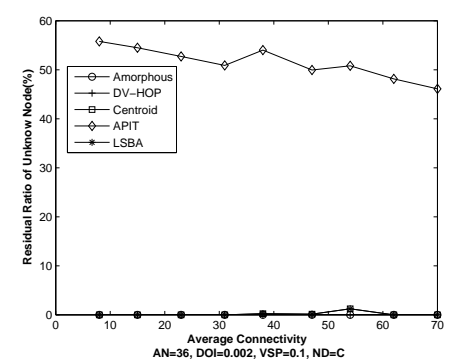

(b) Residual Ratio

Fig. 8. Performance in C-Shaped Area

In fig.8(b), the estimation error of Centroid, LSBA and APIT hardly change when AC increases, meanwhile LSBA and APIT have the lowest estimation error. The estimation error of DV-HOP and Amorphous increase quickly at first and then stay at high level afterwards. The reason is that in both algorithms accurate estimation of Hop-Size depends on both high connectivity and uniformly deployment of nodes, yet the last condition cannot be met in C-shaped area. Moreover, more participant nodes may add more estimated errors in C-shaped area, that's why DV-HOP and Amorphous suffer from high estimation error when average connectivity increases. It seems that DV-HOP and Amorphous are not suitable to work in highly irregular regions.

\section{Conclusion}

In this paper, we provide the motivation, description, and implementation of LSBA, a coarse-grained RSS-based localization algorithm, and the comprehensive performance evaluation of LSBA and four other typical coarse-grained algorithms: Centroid, APIT, DVHOP and Amorphous. Simulation results demonstrate that LSBA achieves the best cost-performance ratio in networks with moderate number of anchors, and moreover LSBA has good adaptability to irregular node deployments. 


\section{References}

1. D. Niculescu and B.Nath. Ad Hoc Positioning System. In: Proceedings of the IEEE Global Communications Conference, November 2001.

2. N. Bulusu et al. GPS-less Low Cost Outdoor Localization for Very Small Devices. IEEE Personal Communications Magazine, 2000.

3. T. He et al. Range-Free Localization Schemes for Large Scale Sensor Networks. In: Proceedings of Annual International Conference on Mobile Computing and Networking, September 2003.

4. D. Niculescu and B.Nath. DV Based Positioning in Ad hocNetworks. Journal of Telecommunication Systems, 2003.

5. R. Nagpal et al. Organizing a Global Coordinate System from Local Information on an Ad Hoc Sensor Network. In: Proceedings of IPSN, 2003.

6. L.Doherty et al. Convex Position Estimation in Wireless Sensor Networks. In: Proceedings of IEEE INFOCOM, April 2001.

7. P. Bahl and V. N. Padmanabhan.RADAR: An In-Building RF-Based User Location and Tracking System. In: Proceedings of the IEEE INFOCOM, March 2000.

8. A. Savvides et al. Dynamic Fine Grained Localization in Ad-Hoc Sensor Networks. In: Proceedings of 7th Annual International Conference on Mobile Computing and Networking, July 2001.

9. K. Chintalapudi et al. Localization Using Ranging and Sectoring. In: Proceedings of IEEE INFOCOM, March 2004.

10. D. Niculescu et al. Ad Hoc Positioning System Using AOA. In: Proceedings of IEEE INFOCOM, April 2003.

11. J. Hightower et al. SpotON: An indoor 3D Location Sensing Technology Based on RF Signal Strength. University of Washington CSE Report, 2000.

12. Kusy. B et al. Node-density independent localization. In: Proceedings of IPSN, 2006.

13. D. Ganesan et al. Complex Behavior at Scale: An Experimental Study of LowPower Wireless Sensor Networks. Technical Report UCLA/CSD-TR 02-0013, 2002.

14. G. Zhou et al. Impact of Radio Irregularity on Wireless Sensor Networks. In: Proceedings of 2nd International Conference on Mobile Systems, Applications, and Services, June 2004.

15. D.Lymberopoulos et al. An Empirical Analysis of Radio Signal Strength Variability in IEEE 802.15.4 Networks using Monopole Antennas, ENALAB Technical Report, Yale University, USA.

16. T.Rappaport et al. Wireless Communications: Principles and Practice. Prentice Hall, 2002.

17. Xin Li, Bei Hua, Yan Guo. Link State Based Annulus Localization Algorithm for Wireless Sensor Networks. In: Proceedings of the International Conference on Communications and Networking in China, 2007. 